\title{
Pathophysiology of HIV related thrombocytopenia: an analysis of 41 patients
}

\author{
A Domínguez, G Gamallo, R Garcia, A Lopez-Pastor, J M Peña, J J Vazquez
}

\begin{abstract}
Aim-To analyse the pathogenic mechanism of HIV related thrombocytopenia. Methods-Forty one patients with thrombocytopenia and HIV-1 infection were investigated over two years. Anticardiolipin antibodies were measured using an enzyme linked immunosorbent assay and antiplatelet antibodies were measured using an immunocapture technique. Tests for VDRL, C3 and C4, antinuclear antibodies and rheumatoid factor were also carried out in all patients and 80 control subjects (HIV-1 positive but non-thrombocytopenic). Indiumoxine labelled platelets were transfused in 13 patients. P24 antigen were also mea-
\end{abstract} sured in 12 bone marrow aspirates.

Results-Antiplatelet antibodies and circulating immune complexes were found exclusively in the thrombocytopenic group; values for antiplatelet antibodies and circulating immune complexes were both higher in homosexual and bisexual patients. Three kinds of pattern were observed using 111 In-labelled platelets: splenic $(n=10)$; hepatic $(n=2)$; and destruction of bone marrow in just one case. The two most influential factors in the sequestration pattern were antiplatelet antibodies in the splenic uptake and circulating immune complexes in the hepatic and marrow sequestration. All patients, except three, had decreased platelet recovery. In those patients with a CD4 lymphocyte count of less than $200 \times 10^{6}$ cells $/$ the recovery was clearly greater $(53 \%)$ than in patients who had more than $200 \times 10^{6}$ in $(28 \%)$. Finally, in seven of the 12 patients who were chosen for immunohistochemical study, p24 antigen was detected in the megakaryocytes, verifying that HIV-1 infects such cells.

Conclusions-The pathogenic mechanism of HIV related thrombocytopenia is probably multifaceted. Antiplatelet antibodies and circulating immune complexes would cause peripheral destruction in the spleen, liver, and bone marrow, in that order; and, on the other hand, there would be an ineffective immune thrombopoiesis and direct infection of the megakaryocytes which could cause a change in the function and maturity of these cells.

(F Clin Pathol 1994;47:999-1003)
Thrombocytopenia is one of the earliest haematological disorders which appears in the evolution of HIV-1 infection. Its association with this infection seems nowadays unquestionable; but its pathophysiological mechanism has not yet been completely defined.

Studies have dealt with the mechanism through which HIV-1 might induce the synthesis of antibodies against the platelets (Ris J, et al. Abstract presented at the XXth Congress of Internal Medicine, Stockholm, 1990. $)^{1-3}$ The polyclonal activation of B lymphocytes could contribute to the synthesis of antibodies and the formation of circulating immune complexes that could be bound on to the platelet membrane. ${ }^{4}$ Experimentally, this mechanism has been related to the thrombocytopenia produced by certain retroviruses.

The presence of circulating immune complexes in HIV-1 positive patients was found in $88 \%$ of cases. $^{5}$ The detection of retroviral antigens in some of the circulating immune complexes proves that the immune system reacts against the virus. ${ }^{6}$ Furthermore, $\mathrm{Yu}$ et $a l^{6}$ have suggested that immunoglobulin might be directed against the anti-HIV antibodies. There are, however, other unresolved issues: among them, the kind of patterns of platelet sequestration in these patients compared with those patients with classic idiopathic thrombocytopenic purpura (ITP), and whether HIV-1 has a more direct association with thrombocytopenia.

\section{Methods}

The study was carried out between 1988 and 1991 at the Internal Medicine Department of "La Paz" Hospital in Madrid (Spain). Forty one patients who met the following criteria were selected: HIV-1 seropositive; a platelet count of less than $100 \times 10^{9} / 1$; and an absence of myelodysplasia, aplastic anaemia, hypersplenism, drug intake, and other viruses.

Thirty two patients were men; nine women. Thirty two were intravenous drug misusers, five were homosexual men, three were heterosexual women and one was a man of unknown risk. At the time of diagnosis, seven patients belonged to the Centers for Disease Control ${ }^{7}$ (CDC) group IV-C1 (AIDS), three to group IV-C2, one to group IV-A, and the other 30 patients to group IV-E. Only those patients with thrombocytopenia alone were included in group IV-E, as we have recommended in previous publications. ${ }^{89}$

Anticardiolipin antibodies (ACA) were measured by enzyme linked immunosorbent 
assay (ELISA) ${ }^{10}$ : in our hospital, the test is considered positive if IgG is $>5 \mathrm{U} / \mathrm{ml}$ or IgM is $>3.2 \mathrm{U} / \mathrm{ml}$, or both. Circulating immune complexes were measured using the Ortho Serum ELISA Test System (C1q-IgG), ${ }^{5}$ regarding as positive those values above 35 $\mu \mathrm{g} / \mathrm{ml}$. Antiplatelet antibodies were analysed by the immunocapture technique (Capture-P. Immucor Inc, Norcross, USA), modified in accordance with procedures published by Juj et $a l^{11}$ and Rachel et al. ${ }^{12}$ The VDRL test and measurement of complement C3 and C4, antinuclear antibodies, and rheumatoid factor were also done. All these variables were also analysed in a control group of $80 \mathrm{HIV}-1$ infected, but non-thrombocytopenic patients.

The transfusion of 111 Indium-oxine (111In) labelled platelets was carried out on 13 patients. All these patients had platelet counts of less than $10 \times 10^{9} / 1$ and none of them was receiving any other treatment. To obtain a greater volume of platelets, and with the purpose of obtaining better results with this test, the studies were carried out with 111In labelled platelets from ABO and HLADR compatible donors, who were hepatitis $B$ surface antigen (HBsAg) and HIV negative. Once the platelets had been labelled, they were administered intravenously. During the first hour blood samples were taken to detect the activity of $111 \mathrm{In}$ and to calculate the percentage of recovery. For an average adult the percentages oscillate between 55 and $72 \% .^{13}{ }^{14}$ After 24 hours, scintigraphic images were obtained using a gamma camera. We investigated three areas: the spleen; the liver; and the thorax-sternum (bone marrow activity). ${ }^{15}$

Finally, to detect HIV-1 infection in megakaryocytes, specific monoclonal antibodies were used against p24 antigen (Chemicom, USA). The study was carried out on 12 biopsy specimens of bone marrow fixed in B5 and paraffin wax, using the avidin biotin alkaline phosphatase technique. The technique had already been applied to samples of lymph nodes from six patients with AIDS (the presence of p24 antigen in endothelial cells, macrophages, and B lymphocytes was observed). To avoid the possibility of false positive results, the same technique was also applied to six samples of bone marrow from HIV negative patients belonging to no behavioural risk group, and here the test did not give any positive result.

The data processing and analysis were carried out on a IBM compatible computer. The graphics were produced with the Harvard Graphics program (Software Publishing Corporation, Mountain View, California USA). The database used was from the SIGMA statistical program (Horus Hardware SA, Madrid, Spain), with which a descriptive study and a comparative analysis of the different variables was performed.

Statistical analysis was performed using Student's $t$ test and $\chi^{2}$ analysis, with Yates's correction. Values of $p<0.05$ were regarded as significant.

\section{Results}

The results of the different immunological variables relating thrombocytopenic and nonthrombocytopenic patients are shown in table 1. Positive circulating immune complexes were present in $64.1 \%$ of the thrombocytopenic patients and in $17.7 \%$ of the nonthrombocytopenic patients; $\mathrm{p}<0.05$. Antiplatelet antibodies and circulating immune complexes were found exclusively in the thrombocytopenic group. There were no significant differences between the two groups with the rest of the immunological data. The antiplatelet antibody and circulating immune complexe values in the different risk groups are shown in table 2 . The most significant difference $(p<0.05)$ was the circulating immune complex percentage in homosexual men $(80 \% ; 4 / 5)$ compared with the rest of the risk groups $(23.52 \% ; 8 / 34)$. There were no measurable differences between the values of anticardiolipin IgG and IgM antibodies in either group (table 3).

Table 4 gives details of the patients who were subjected to a gamma camera study to detect the areas of greatest platelet destruction. Three main patterns were observed: (1) splenic ( $n=10)$; one patient (case 10) also showed increased hepatic uptake; (2) hepatic $(n=2)$ (cases 9 and 11): both showed simultaneous increases in uptake in bone marrow;

Table 1 Immunological variables in thrombocytopenic and non-thrombocytopenic patients

\begin{tabular}{llll}
\hline & $\begin{array}{l}\text { Thrombocytopenic } \\
(n=41)\end{array}$ & $\begin{array}{l}\text { Non- } \\
\text { thrombocytopenic } \\
(n=80)\end{array}$ & p value \\
\hline RF & $17 \cdot 85 \%(5 / 28)$ & $9 \cdot 52 \%(4 / 42)$ & $0 \cdot 7259$ \\
ANA & $0 \%(0 / 28)$ & $0 \%(0 / 42)$ & \\
VDRL & $12 \cdot 5 \%(5 / 40)$ & $36 \cdot 58 \%(15 / 41)$ & $0 \cdot 4376$ \\
ACA IgG & $44 \cdot 73 \%(17 / 38)$ & $72 \cdot 41 \%(42 / 58)$ & 0.5891 \\
ACA IgM & $18 \cdot 42 \%(7 / 38)$ & $43 \cdot 10 \%(25 / 58)$ & $0 \cdot 4027$ \\
C3 (mg/dl) & 96.63 & $98 \cdot 16$ & $0 \cdot 8813$ \\
C4 (mg/dl) & $38 \cdot 95$ & 33.94 & 0.6603 \\
CIC & $30 \cdot 76 \%(12 / 39)$ & $0 \%(0 / 45)$ & $0.0000^{\star}$ \\
ApAb & $56.41 \%(22 / 39)$ & $0 \%(0 / 38)$ & $0.0000^{\star}$
\end{tabular}

$\mathrm{RF}(+)=$ Rheumatoid factor; ANA = antinuclear antibodies ACA = anticardiolipin antibodies; CIC = circulating immune complexes; $\mathrm{ApAB}=$ antiplatelet antibodies.

$\star$ Significant differences.

Table 2 Percentage of antiplatelet antibodies and circulating immune complexes

\begin{tabular}{lllr}
\hline & $\begin{array}{l}\text { Homosexual } \\
\text { and } \\
\text { bisexual men }\end{array}$ & $\begin{array}{l}\text { Drug } \\
\text { misusers }\end{array}$ & Others \\
\hline $\begin{array}{l}\text { Circulating immune } \\
\begin{array}{c}\text { complexes } \\
\text { Antiplatelet antibodies }\end{array}\end{array}$ & $80 \%$ & $23 \%$ & $33 \%$ \\
\hline
\end{tabular}

*The difference was significant $(p<0.05)$ between drug

misusers and homosexual or bisexual patients.

Table 3 Percentage of anticardiolipin antibodies in thrombocytopenic and non-thrombocytopenic patient

\begin{tabular}{lll}
\hline $\begin{array}{l}\text { Anticardiolipin } \\
\text { antibodies }\end{array}$ & Thrombocytopenic & $\begin{array}{l}\text { Non- } \\
\text { thrombocytopenic }\end{array}$ \\
\hline IgG & $\mathbf{4 4 \cdot 7 4 \%}$ & $\mathbf{7 2 . 4 1 \%}$ \\
IgM & $18 \cdot 42 \%$ & $43 \cdot 11 \%$ \\
\hline
\end{tabular}

The differences were not significant. 
Table 4 Distribution of Indium labelled platelets in 13 patients with HIV related thrombocytopenia

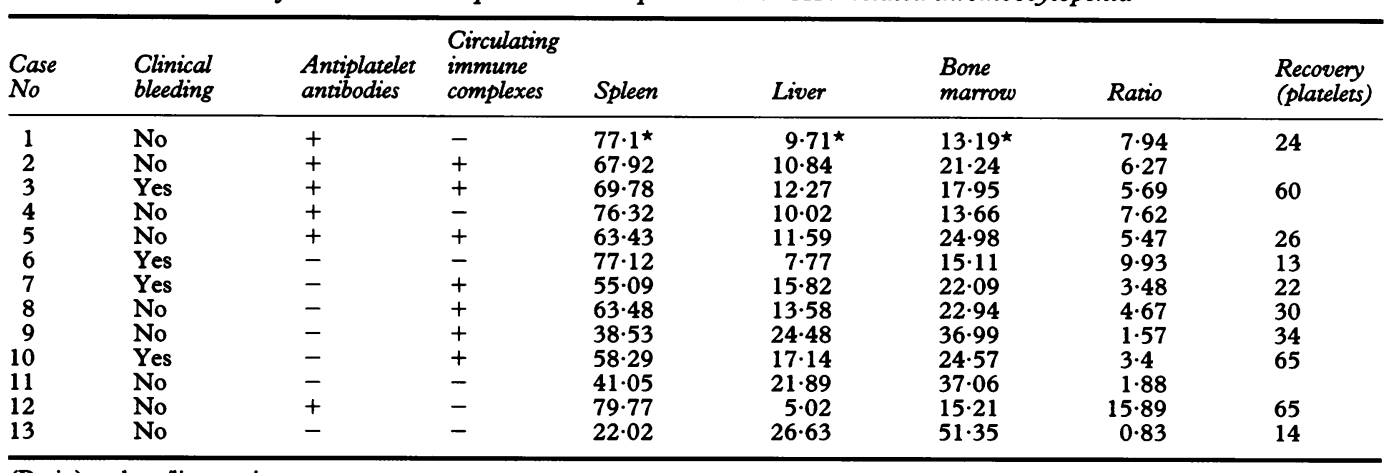

(Ratio): spleen/liver ratio.

* Percentage of activity in spleen, liver, and bone marrow.

Table 5 Spleen: liver ratio ${ }^{*}$ in patients with HIV related thrombocytopenia

\begin{tabular}{lll}
\hline Variable & Ratio & p value \\
\hline Drug misusers: & $4 \cdot 87$ & \\
Other risk groups & $7 \cdot 13$ & NS \\
Antiplatelet antibodies (+) & $8 \cdot 14$ & \\
Antiplatelet antibodies (-) & $4 \cdot 16$ & $<0.05$ \\
Circulating immune complexes (+) & $4 \cdot 13$ & \\
Circulating immune complexes (-) & $8 \cdot 65$ & $<0.05$ \\
CD4 <200: & $4 \cdot 57$ & \\
CD4 > 200 & $6 \cdot 26$ & NS \\
$<50 \times 10^{9} /$ platelets: & $4 \cdot 46$ & \\
$>50 \times 10^{\circ} /$ platelets & $7 \cdot 79$ & NS \\
Clinical bleeding & $5 \cdot 62$ & \\
No symptoms & $5 \cdot 79$ & NS
\end{tabular}

*Spleen/liver ratio of distribution of Indium labelled platelets; $\mathrm{ApAb}=$ antiplatelets antibodies; $\mathrm{CIC}=$ circulating immunocomplexes; CD4 = lymphocytes $/ \mathrm{mm}^{3}$. NS = not significant.

(3) bone marrow pattern $(n=1)$ (case 13, who also showed increased hepatic uptake). The ratio between hepatic and splenic uptake decreased in three cases, remained stable in three, and increased in seven (in case 12 this even reached $15 \cdot 89)$. Platelet recovery decreased in all except three cases (7/10).

Patients with antiplatelet antibodies titres had greater splenic uptake than those negative for antiplatelet antibodies, although in these patients hepatic and bone marrow uptakes were greater $(p<0.05)$. For circulating immune complexes, it was exactly the opposite pattern (table 5). There were no differences, however, between patients from different risk groups; between patients who did or did not experience clinical bleeding; between those patients who had more or less than $50 \times 10^{9} / 1$ platelets, nor even between those who had more or less than $200 \times 10^{6}$ lymphocytes CD4/l. The recovery was $54.01 \%$ in those who had a CD4 lymphocyte count of less than $200 \times 10^{6} / 1$, compared with $27.71 \%$ for those who had more than $200 \times 10^{6} / 1(\mathrm{p}<0.01)$.

P24 antigen was found in the membrane and cytoplasm of megakaryocytes and cells of the myeloid-lymphoid series in seven of 12 cases studied.

\section{Discussion}

Important immunological changes occur in HIV infection. Okenhendler (Abstract presented to Sixth International Conference on AIDS, San Francisco, 1990) and Karpatkin ${ }^{16}$ have suggested that anti-idiotype antibodies
anti-HIV antibodies or antibodies to unknown platelet antigens might be responsible.

We compared the findings in thrombocytopenic patients with those found in HIV-1 positive but non-thrombocytopenic patients. Antiplatelet antibodies and circulating immune complexes were exclusive to the thrombocytopenic group (56 and $31 \%$, respectively). The rest of the analysed variables did not show any significant differences between the thrombocytopenic and the nonthrombocytopenic patients.

The presence of antiplatelet antibodies could be related to a secondary immune alteration caused by an increase in the polyclonal activity of B lymphocytes caused by HIV. This would stimulate the production of crossreacting antibodies, ${ }^{17}$ autoantibodies, and antibodies as opposed to viral adsorbed antigens or any other unknown antigens. The discovery of circulating immune complexes in HIV related thrombocytopenia coincides with what has been described in classic secondary thrombocytopenias (drugs or other viral infections). ${ }^{18}$ Antiplatelet antibodies and circulating immune complexes were more common in the homosexual and bisexual men than in the drug misusers, though circulating immune complexes were significantly greater $(p<$ $0.05)$ in the former group ( $80 \%)$ than in the drug misuser group (23\%). Our findings seem to confirm Karpatkin's findings ${ }^{19-21}$ concerning the connection between infection with the virus and different pathogenic mechanisms. However, there is no obvious explanation for the higher amounts of circulating immune complexes in homosexual men.

Finally, anticardiolipin antibodies, IgG, and $\operatorname{IgM}$ do not seem to be related to the pathogenesis of thrombocytopenia, in contrast to the findings of Harris ${ }^{10}$ concerning classic ITP. Like Intrator $e t a l,{ }^{22}$ we have not found any such association.

Thrombocytopenia is probably produced by a combination of marrow destruction, with subsequent ineffective production of, and a decrease in, the number of circulating platelets due to the sequestration and destruction caused by some of the peripheral organs of the mononuclear phagocytic system. ${ }^{23}$

Kinetic studies of $111 \mathrm{In}$ in patients with classic ITP show three kinds of pattern: 
destruction in the spleen, the liver, and bone marrow..$^{1524}$ These patterns were also observed in our study. The significant increase in the destructive activity of the spleen in HIV positive patients was interesting and was considerably greater than in patients with classic ITP. This could explain the excellent results obtained by removing the spleen of these patients, although opinions vary as to the correlation between the results of the 111In labelled platelet studies and the therapeutic response. ${ }^{1315}$

Nearly all of the patients had a low platelet recovery, which shows a rapid decrease in transfused platelets as a result of peripheral destruction. ${ }^{15}$ Measurable differences $(p<$ 0.01 ) were only found in patients with less than $200 \times 10^{6} \mathrm{CD} 4 / 1$ lymphocytes who showed a recovery of $53 \%$ (practically normal) compared with $28 \%$ (decreased) in the others. In fact, patients from the IV-E group, with a better immunological state, showed, during the whole study, a lower platelet count than those from the IV-C1 group, with a low CD4 count and a possible macrophagic disorder, which might explain why peripheral destruction was not so great in these patients.

There were no differences in clinical bleeding or platelet count among the behavioural risk groups, but we found important differences in the presence of antiplatelet antibodies or circulating immune complexes. There was an increase in splenic uptake for antiplatelet antibody positive patients and in hepatic and marrow uptake for circulating immune complex positive patients. The exact cause for the presence of circulating immune complexes or antiplatelet antibodies which influences the kind of sequestration pattern is not known. A possible explanation could be the existence of a different platelet "affinity" in the different sequestration levels. Liver and bone marrow, with cells containing receptors for IgM and C3, probably have a more important role in the elimination of platelets in patients whose platelets have circulating immune complexes bound on to the surface,

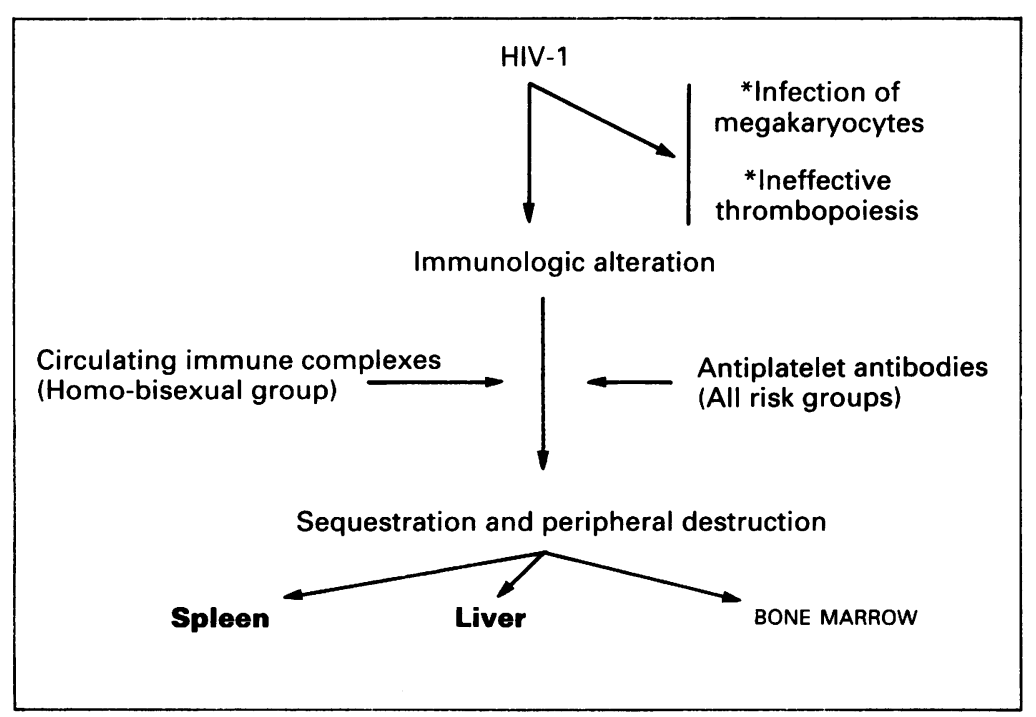

Pathogenic hypothesis of HIV related thrombocytopenia. while the antibody coated platelets would be more likely to be phagocytosed by the macrophages of the spleen as happens in classic ITP.

The importance of the bone marrow as a sequestration area is possibly greater than has been found in kinetic platelet studies. ${ }^{15}$ On the other hand, some authors suggest that HIV infected megakaryocytes could inhibit platelet production. ${ }^{25}$ There is also evidence of megakaryocytic growth being affected by this viral infection, as well as morphological changes produced in such cells. ${ }^{26}$ But these studies were done in vitro. As far as we are aware there is no in vivo evidence for this as yet.

To detect the presence of $\mathrm{p} 24$ antigen, and therefore $\mathrm{HIV}$, in the bone marrow we used a monoclonal antibody technique that has previously been used to detect such antigens in the lymphocytes of the lymph nodes or other cells - for example in the intestine. ${ }^{27}$ Although only 12 patients were studied, we managed to prove that megakaryocytes were infected with HIV-1, by detecting p 24 antigen in cytoplasm and the membrane. There was no direct correlation between the presence of $\mathrm{p} 24$ antigen in blood and the presence or absence of antiplatelet antibodies or circulating immune complexes.

Infection of the megakaryocytes could produce, in some patients, ineffective autoimmune thrombopoiesis similar to that which occurs in classic ITP, ${ }^{28}$ or it could produce a maturative and functional change as a result of direct infection by the virus. The primary mechanism of the virus could be the following: HIV-1 induces the production of thrombopoiesis inhibitors or penetrates megakaryocytes through a receptor that has as yet not been identified. This receptor could be $\mathrm{CD} 4{ }^{28}$ another unidentified antigen, or could even be introduced by the circulating immune complexes and used as a "key" through the Fc receptor. The last mechanism has been seen in other virus infections, ${ }^{16}$ but obviously it is not unique as not all the patients showing p24 antigen in megakaryocytes were positive for circulating immune complexes.

In conclusion, HIV-1 related thrombocytopenia could have a multifaceted pathogenic mechanism (figure). Antiplatelet antibodies and circulating immune complexes could cause the consequent peripheral destruction in the spleen, liver, and bone marrow in that order; and, on the other hand, there would be ineffective immune thrombopoiesis and direct infection of the megakaryocytes, resulting in a change in the function and maturity of these cells.

1 Ribera A, Perez P, Lima J, Monteagudo M, Bastida MT. Anticuerpos antiplaquetas en pacientes adictos a drogas por via parenteral [abstract]. Sangre (Barc) 1986;31:65 2 Stricker RB, Abrams DI, Curash L, Shuman MA. Target platelet antigen in homosexual men with immune thrombocytopenia. $N$ Engl $f$ Med 1985;313:1375-80.

3 Van der Lelie J, Lange JM, Vos JJ, van Dalen CM, Danner $\mathrm{SA}$, von dem Borne AE. Autoimmunity against blood cells in human immunodeficiency virus infection. $B r \mathcal{F}$ Haematol 1987;67:109-14. 
4 Lima J. Plaquetas y VIH. Med Clin (Barc) 1989;92:655-7. 5 Morrow WJ, Wharton M, Stricker RB, Levy JA. Circulating immunecomplexes in patients with AIDS contain the AIDS-associated retrovirus. Clin Immunol Immunopathol 1986;40:515-24.

6 Yu JR, Lennette ET, Karpatkin S. Anti-F(ab') 2 antibodies in thrombocytopenia patients at risk for AIDS. $\mathcal{f}$ Clin Invest 1986;77:1756-61.

7 Centers for Disease Control (CDC). Classification system for human T-lymphotropic virus type III/lymphadenopathy-associated virus infections. $M M W R$ 1986;35: 324-39.

8 Dominguez A, Peña JM, Del Arco A, Vazquez JJ. Thrombocitopenia inmune asociada a la infeccion por el VIH. Interrogantes patogenicos, terapeuticos y conceptuales. Med Clin (Barc) 1990;94:519.

9 Dominguez A, Martinez MA, Peña JM, Vazquez JI. HIVassociated immune thrombocytopenia. Lancet 1989;ii: 459.

10 Harris EN, Gharavi UH, Derue G, Morgan SH Anticardiolipin antibodies in autoimmune thromboAnticardiolipin antibodies in autoimmune throm

11 Juji T, Kano K, Milgrom F. Mixed agglutination with platelets. Int Arch Allergy Immunol 1972;42:474.

12 Rachel JM, Sinor LT, Tawfik OW. A solid-phase red cell adherence test for platelet crossmatching. Med Lab Sci 1985;42:194.

13 Castle VP, Shulkin BL, Coates G, Andrew M. The use of Indium-111 oxine platelet scintigraphy and survival studies in pediatric patients with thrombocytopenia. Ұ Nucl Med 1989;30:1819-24.

14 Heyns AP, Lötter MG, Badenhorst PN, van Reenen OR, Pieters H, Minnaar PC, et al. Kinetics, distribution and sites of destruction of 111 Indium-labelled human sites of destruction of 111 Indium-lab

15 platelets. Broters $\mathrm{H}$, Herbst $\mathrm{C}$, et al. Kinetics and sites of destruction of 111 Indium-oxine-labelled platelets in idiopathic thrombocytopenic purpura. Am $\mathcal{f}$ Hematol 1982;12:167-77.

16 Karpatkin S. HIV-1 related thrombocytopenia. Hematol Oncol Clin North Am 1990;4:193-218.
17 Murphy MF, Metcalfe P, Waters AH, Carne CA, Weller IV, Linch $C$, et al. Incidence and mechanism of neutropenia in patients with HIV infection. Br 7 Haematol 1987;66:337-40.

18 McMillan R. Chronic idiopathic thrombocytopenic purpura. N Engl f Med 1981;304:1135-47.

19 Savona S, Nardi MA, Lennette ET, Karpatkin S Thrombocytopenic purpura in narcotics addicts. Ann

20 Walsh CM, Nardi MA, Karpatkin S. On the mechanism of thrombocytopenic purpura in sexually active homosexual men. $N$ Engl $\mathfrak{f}$ Med 1984;311:635-9.

21 Walsh CM, Karpatkin S. Thrombocytopenia and HIV-1 infection. Semin Oncol 1990;17:367-74.

22 Intrator L, Oksenhendler E, Desforges L, Bierling $P$ Anticardiolipin antibodies in HIV infected patients with or without immune thrombocytopenic purpura. $\mathrm{Br} \mathscr{f}$ Haematol 1988;68:269-70.

23 Gernsheimer T, Stratton J, Ballem PJ, Slichter SJ Mechanism of response to treatment in autoimmune Mechanism of response to treatment in autoimmune
thrombocytopenic purpura. $N$ Engl $₹$ Med 1989;320: 974-80.

24 International Committee for Standardization in Hematology Panel on Diagnosis applications of radionuclides. Recommended method for Indium-111 platelet survival studies. F $\mathrm{Nucl} \mathrm{Med} \mathrm{1988;29:564-6.}$

25 Stella CC, Bergamaschi G, Nalli G, Cazzola M. "In vitro" megakaryocytopoiesis in patients with HIV-related thrombocytopenic purpura. Haematologica 1988;73: 25-30.

26 Gernsheimer T, Stratton J, Ballen PJ, Slichter SJ Treatment of autoimmune thrombocytopenia purpura. $N$ Engl $\tilde{F}$ Med 1989;321:761.

27 Ullrich R, Zeitz $M$, Heise W, L'age $M$, Höffken G, Riecken EO. Small intestinal structure and function in Riecken EO. Small intestinal structure and function in enteropathy. Ann Intern Med 1989;111:15-21.

28 Mintzer DM, Real FX, Jovino L, Krown SE. Treatment of Kaposi's sarcoma and thrombocytopenia with vincristine in patients with AIDS. Ann Intern Med 1985, 102:200-2. 